Short Communication

\title{
Association of antimicrobial usage with faecal abundance of aph(3')-III, erm $B$, sul2 and tetW resistance genes in veal calves in three European countries
}

\author{
Dongsheng Yang a,*, Liese Van Gompel ${ }^{a}$, Roosmarijn E.C. Luiken ${ }^{a}$, Pim Sanders ${ }^{a}$, \\ Philip Joosten $^{\mathrm{b}}$, Eri van Heijnsbergen ${ }^{\mathrm{a}}$, Inge M. Wouters ${ }^{\mathrm{a}}$, Peter Scherpenisse ${ }^{\mathrm{a}}$, \\ Claire Chauvin ${ }^{c}$, Katharina Wadepohl ${ }^{\mathrm{d}}$, Gerdit D. Greve ${ }^{\mathrm{a}}$, \\ Betty G.M. Jongerius-Gortemaker ${ }^{a}$, Monique H.G. Tersteeg-Zijderveld ${ }^{a}$, \\ Christophe Soumet ${ }^{\mathrm{c}}$, Magdalena Skarżyńska ${ }^{\mathrm{e}}$, Katharina Juraschek ${ }^{\mathrm{f}}$, Jennie Fischer ${ }^{\mathrm{f}}$, \\ Dick J.J. Heederik ${ }^{a}$, Lidwien A.M. Smit ${ }^{\mathrm{a}}$, on behalf of the EFFORT consortium

\footnotetext{
${ }^{\mathrm{b}}$ Veterinary Epidemiology Unit, Faculty of Veterinary Medicine, Ghent University, Ghent, Belgium

${ }^{c}$ ANSES, Epidemiology, Health and Welfare Unit, Paris, France

${ }^{\mathrm{d}}$ Außenstelle für Epidemiologie, Tierärztliche Hochschule Hannover, Hannover, Germany

e Department of Microbiology, National Veterinary Research Institute, Pulawy, Poland

${ }^{\mathrm{f}}$ Department of Biological Safety, German Federal Institute for Risk Assessment, Berlin, Germany

${ }^{g}$ Department of Infectious Diseases and Immunology, Utrecht University, Utrecht, The Netherlands

${ }^{\mathrm{h}}$ National Institute for Public Health and the Environment, Bilthoven, The Netherlands

i Department of Bacteriology and Epidemiology, Wageningen Bioveterinary Research, Lelystad, The Netherlands
} \\ anstitute for Risk Assessment Sciences, Faculty of Veterinary Medicine, Utrecht University, Utrecht, The Netherlands
} Dariusz Wasyl ${ }^{\mathrm{e}}$, Jaap A. Wagenaar ${ }^{\mathrm{g}, \mathrm{i}}$, Jeroen Dewulf ${ }^{\mathrm{b}}$, Heike Schmitt ${ }^{\mathrm{a}, \mathrm{h}}$, Dik J. Mevius ${ }^{\mathrm{g}, \mathrm{i}}$,

\section{A R T I C L E I N F O}

\section{Article history:}

Received 7 November 2019

Accepted 29 July 2020

\section{Keywords:}

Antimicrobial resistance

Resistance genes

qPCR

Risk factors

Veal calves

\begin{abstract}
A B S T R A C T
Background: High antimicrobial use (AMU) and antimicrobial resistance (AMR) in veal calves remain a source of concern. As part of the EFFORT project, the association between AMU and the abundance of faecal antimicrobial resistance genes (ARGs) in veal calves in three European countries was determined.

Methods: In 2015, faecal samples of veal calves close to slaughter were collected from farms located in France, Germany and the Netherlands (20 farms in France, 20 farms in the Netherlands and 21 farms in Germany; 25 calves per farm). Standardized questionnaires were used to record AMU and farm characteristics. In total, 405 faecal samples were selected for DNA extraction and quantitative polymerase chain reaction to quantify the abundance (16S normalized concentration) of four ARGs [aph(3')-III, ermB, sul2 and tetW] encoding for resistance to frequently used antimicrobials in veal calves. Multiple linear mixed models with random effects for country and farm were used to relate ARGs to AMU and farm characteristics.

Results: A significant positive association was found between the use of trimethoprim/sulfonamides and the concentration of sul2 in faeces from veal calves. A higher weight of calves on arrival at the farm was negatively associated with $\operatorname{aph}\left(3^{\prime}\right)-I I I$ and ermB. Lower concentrations of aph(3')-III were found at farms with non-commercial animals present. Furthermore, farms using only water for the cleaning of stables had a significantly lower abundance of faecal ermB and tetW compared with other farms.
\end{abstract}

\footnotetext{
* Corresponding author. Yalelaan 2, 3584CM Utrecht, The Netherlands. Tel.: +31 302532489.

E-mail address: d.yang@uu.nl (D. Yang).
} 
Conclusion: A positive association was found between the use of trimethoprim/sulfonamides and the abundance of sul2 in faeces in veal calves. Additionally, other relevant risk factors associated with ARGs in veal calves were identified, such as weight on arrival at the farm and cleaning practices.

(c) 2020 Elsevier Ltd and International Society of Antimicrobial Chemotherapy. All rights reserved.

\section{Introduction}

The global emergence of antimicrobial resistance (AMR) is considered to be a large threat to human health [1], and has resulted, in part, to antimicrobial use (AMU) in animals [2]. In veal calf farming, high AMU and AMR remain a source of concern. The calf trading network is complex, and calves from different farms are mixed and transported across regional and international borders [3]. Consequently, and because the immune system of a calf is not fully developed until approximately 6 months after birth [4], calves have an increased risk of developing infectious diseases, resulting in high AMU in veal calves [5].

In order to control the level of AMR in veal calves, there is an urgent need to quantify the abundance of antimicrobial resistance genes (ARGs) and to identify the potential risk factors. ARGs can be transferred horizontally between bacterial species. Compared with focusing on pathogenic-resistant bacteria, studying ARGs can provide a more comprehensive overview of AMR in livestock [6]. To determine the potential determinants of AMR in veal calves, a previous study found a positive association between AMR and AMU in veal calves [7]. Interestingly, a longitudinal study has shown that the prevalence of meticillin-resistant Staphylococcus aureus (MRSA) in calves and stable air did not increase simultaneously with or directly after treatment with antimicrobials [8], suggesting that AMU may not be the only determinant for AMR in veal calves.

As part of the Ecology from Farm to Fork of Microbial Drug Resistance and Transmission (EFFORT) project, this study aimed to better understand the relationship between AMU and the abundance of selected ARGs in veal calves. This study investigated the abundance of genes encoding resistance to four antimicrobial classes: aminoglycosides, macrolides, tetracyclines and sulfonamides $[2,6,8,9]$. Antimicrobials that belong to these classes and the combined use of trimethoprim/sulfonamides (trim/sulfa) are the most widely used antimicrobials in European veal calves $[3,6,9]$. Associations between faecal ARGs with the abovementioned antimicrobial classes ( $\operatorname{aph}\left(3^{\prime}\right)$-III, ermB, sul2 and tetW) and potential farm-related risk factors (e.g. AMU, weight of calves and stable cleaning agents) were determined in France, Germany and the Netherlands.

\section{Materials and methods}

\subsection{Study design and study population}

Between January and December 2015, a cross-sectional study was conducted in 61 veal calf farms in France, Germany and the Netherlands. Country names were anonymized to ' $B$ ', ' $E$ ' and ' $F$ ' in line with previous EFFORT publications $[6,10]$. In each of the participating countries, conventional non-mixed white or rosé veal calf farms were visited. Sampling was spread over the entire year. Only individual farms rearing at least 200 animals per production round with no contacts through trade and using an 'all-in-allout' production system were included in the study. Farm selection was based, in part, on convenience (e.g. distance from the research institute to the farms). Therefore, the sampling of farms in each country cannot be considered representative of the entire veal calf sector in that country.

\subsection{Faecal sampling and data collection}

\subsubsection{Faecal sample collection}

At each farm, 25 individual calves were sampled in their last weeks before slaughter. Faecal samples were collected using sterile spoons during or directly after defaecation from faecal parts without floor contact. No animal ethics approval from the respective national authorities was needed (non-invasive faecal sampling process). For each calf, a minimum of $10 \mathrm{~g}$ of faeces was collected in a sterile faeces container. After collection, the samples were stored at $4^{\circ} \mathrm{C}$, transported to the laboratory within $24 \mathrm{~h}$, homogenized and stored at $-80^{\circ} \mathrm{C}$ until DNA extraction.

\subsubsection{Data on $A M U$ and farm characteristics}

A standardized questionnaire was completed by the farmers. This included questions on AMU, farm and herd characteristics, and farm management. AMU group treatment incidences (TIs) based on defined daily doses for animals (DDDvet) were computed at the farm level. More technical details have been described previously [11].

\subsection{DNA extraction and $q P C R$}

Of the 25 faecal samples collected at the farms, seven individual faecal samples from each farm were selected at random for quantitative polymerase chain reaction (qPCR) detection and analysis. DNA was extracted using the modified QIAmp Fast DNA stool mini kit (Cat. No. 51604; Qiagen, Venlo, The Netherlands). Following DNA extraction, qPCR was performed to quantify the relative abundance of ARGs. More details regarding the qPCR assays, quality control steps [internal amplification control (IAC), replicate consistency check] and $16 \mathrm{~S}$ normalization have been described previously (16S, ermB, tetW) [12]. For aph(3')-III and sul2, the qPCR process differed slightly in PCR reaction composition (primers, probe): [aph(3')-III - $400 \mathrm{nM}, 250 \mathrm{nM}$; sul2: $100 \mathrm{nM}, 100 \mathrm{nM}]$ [13,14], and limit of detection/quantification (LOD/LOQ) and qPCR efficiency percentage: $\operatorname{aph}\left(3^{\prime}\right)-I I I-0.22,0.52,101.6 \%$; sul2 - 0.92, 0.91, 93.6\%. All genes were expressed as $\log _{10}$ gene copies.

\subsection{Statistical analysis}

SAS Version 9.4 and R Version 3.6.3 were used for statistical analysis. Potential risk factors were chosen based on the published literature on AMR risk factors in veal calves (age, weight, farm size, season) $[7,15]$ or other livestock sectors (non-commercial animals [16], type of cleaning agents [17]).

After qPCR quality control (IAC and replicate consistency), values below the LOD were removed (16S gene) or replaced [aph(3')III, sul2]. ARGs were replaced with half of the lowest untransformed value $>$ LOD per gene and country before $\log _{10}$ transforming these again.

AMU data were strongly right skewed; therefore, $\log _{10}$ transformation was applied $[\log 10(\mathrm{AMU}+1)]$. To take the between- and within-country variation into account, a linear mixed model with a random effect for both country and farm was applied. 


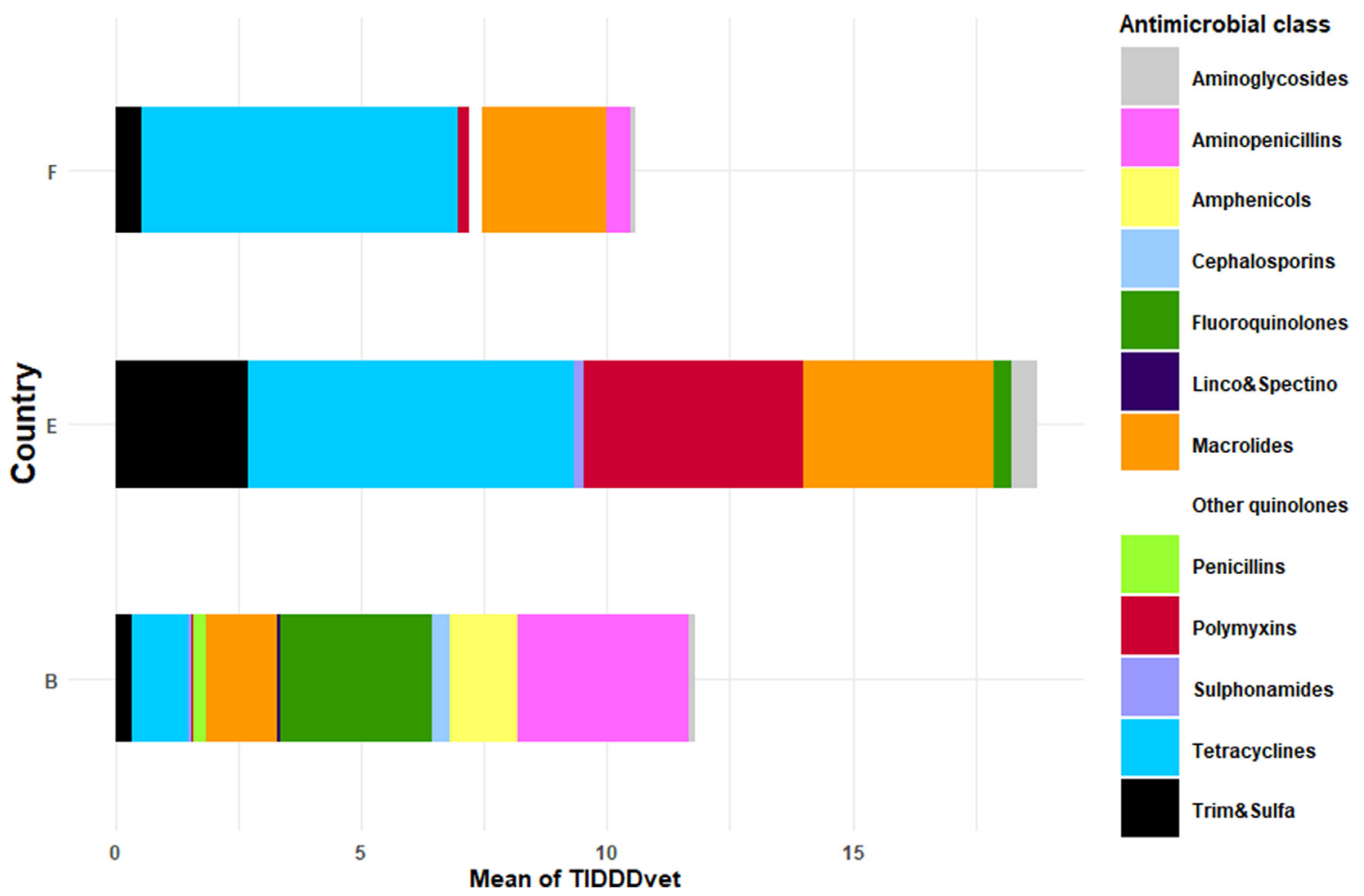

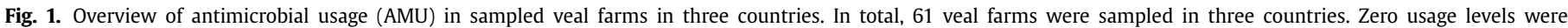

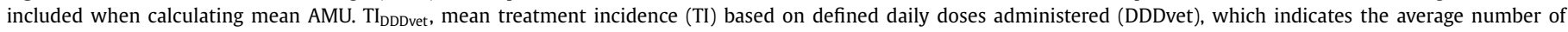
treatment-days per 100 days. Trim, trimethoprim; sulfa, sulfonamides.

AMU and other farm-related factors (fixed effect variables) were first selected in a univariate analysis $(P<0.20)$ and subsequently introduced into a multiple linear mixed model per ARG. Correlations between the included independent variables were checked, and only variables with low correlation were included in the full multivariable model [both continuous variables: Pearson correlation $(\rho)$ $<0.7$; both categorical variables: Chi-squared test $(P>0.05)$; one continuous variable and one categorical variable: one-way analysis of variance (ANOVA) $(P>0.05)]$. Subsequently, the full models (containing all possible risk factors and confounders) were reduced manually, employing a backward selection based on the Akaike information criterion to obtain the final model. To make the model coefficients more interpretable, all coefficients were exponentiated to obtain geometric mean ratios (GMR and 95\% confidence intervals). Before exponentiation, estimates corresponding to numeric determinants were multiplied by the interquartile range (IQR) to obtain the GMR for an interquartile range increase in the determinant.

\section{Results}

\subsection{Antimicrobial use}

The types of antimicrobials and amount of AMU varied across the three countries (Fig. 1). Macrolides (84\%, 60\% and $100 \%$ of farms in Countries B, E and F, respectively) and tetracyclines (84\%, $90 \%$ and $100 \%$ of farms in Countries $\mathrm{B}, \mathrm{E}$ and $\mathrm{F}$, respectively) were widely used. In Country B, aminopenicillins (95\%), amphenicols $(79 \%)$ and fluoroquinolones (89\%) were also widely used. Two farms were excluded from the analysis due to missing qPCR data or because AMU was out of the range of expected values, resulting in 59 farms for data analysis. As only eight of the 59 farms used aminoglycosides, $\mathrm{TI}_{\text {DDDvet }}$ for aminoglycosides was replaced by a binary variable indicating aminoglycoside use (yes/no).

\subsection{Abundance of ARGs}

After qPCR quality control and $16 \mathrm{~S}$ normalization, 124-137, 118137 and 89-131 (min-max) samples per ARG model remained for analysis within Countries B, E and F, respectively. Significant differences were found between the three countries for the mean abundance of all targets [aph(3')-III $(P<0.01)$, sul2 $(P<0.01)$ and tetW $(P=0.04)$; one-way ANOVA], except for $\operatorname{ermB}(P=0.08)$.

\subsection{Associations between farm characteristics and abundance of ARGs}

Fig. 2 illustrates the relationship between the ARGs and AMU for the different antimicrobial classes among the three countries. For each ARG, a final linear mixed model was fitted (Table 1).

A significant positive association was found between trim/sulfa use and the abundance of sul2 in faecal samples from calves $(\mathrm{GMR}=1.37, P<0.01)$. Furthermore, a higher weight of calves on arrival at the farm was negatively associated with faecal aph(3')-III $(\mathrm{GMR}=0.82, P=0.01)$ and ermB $(\mathrm{GMR}=0.72, P<0.01)$ loads. Lower faecal aph(3')-III levels were found in calves at farms where non-commercial animals (e.g. cats, dogs, sheep) were present (GMR=0.71, $P=0.05)$. Veal calves sampled at farms that used only water for cleaning of stables carried lower ermB $(\mathrm{GMR}=0.5$, $P=0.01)$ and tet $W(\mathrm{GMR}=0.66, P=0.04)$ concentrations in their faeces compared with veal calves of farms which used both soaking agents and disinfectants. Agents used for cleaning stables were not included in the final model of $a p h\left(3^{\prime}\right)$-III because of correlation with the use of aminoglycosides $(P<0.01$, Chi-squared test).

\section{Discussion}

This study determined the potential risk factors for faecal carriage of ARGs in veal calves close to slaughter. A significant positive 

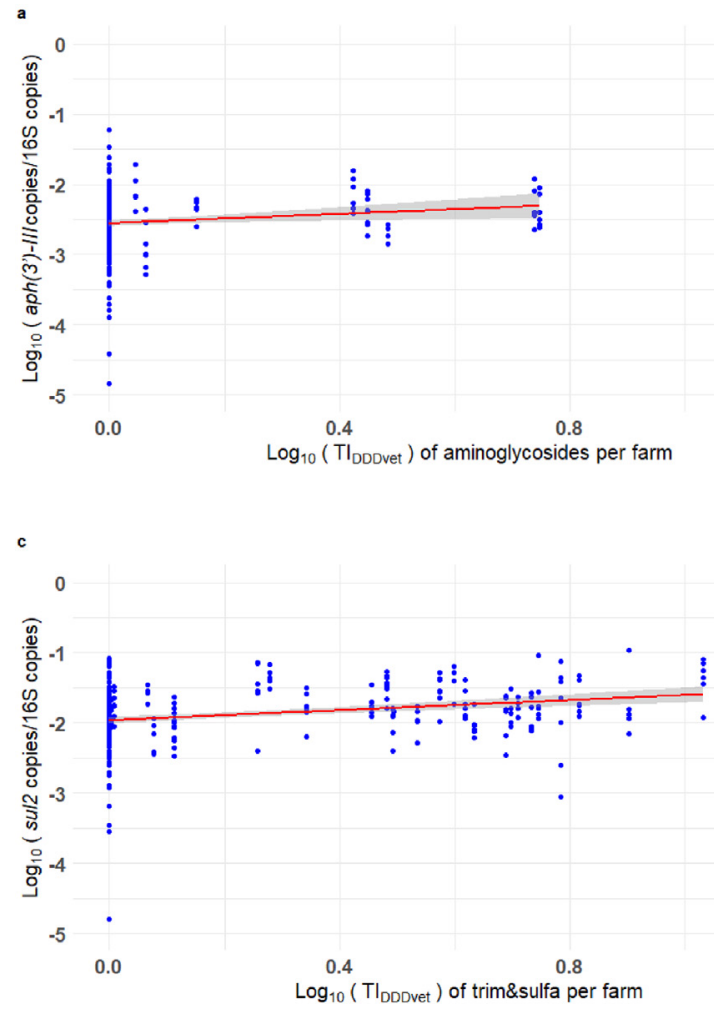

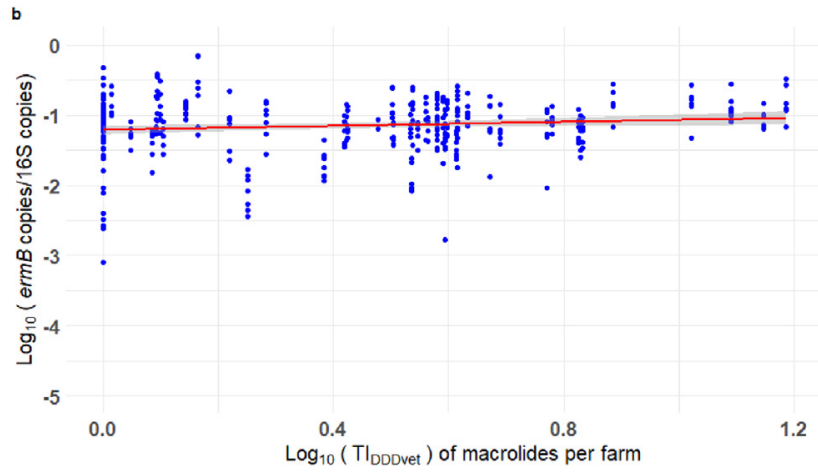

d

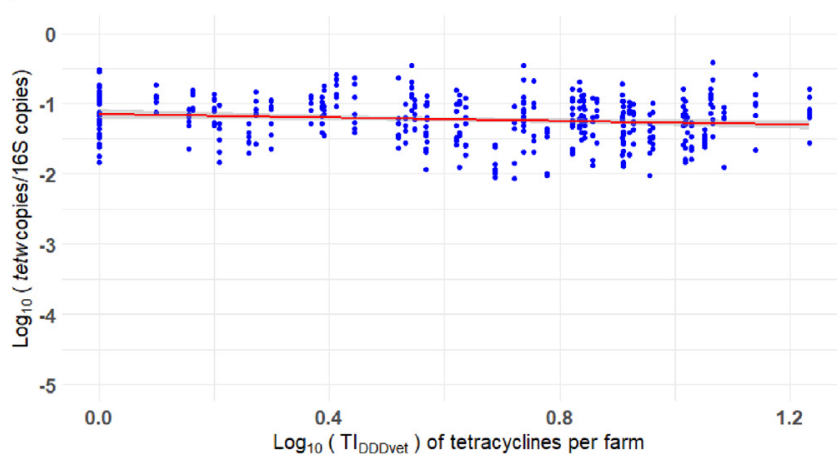

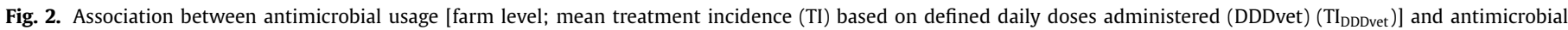

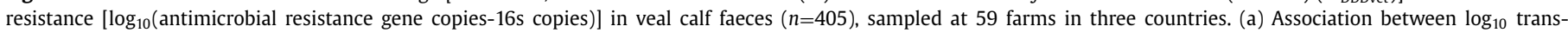

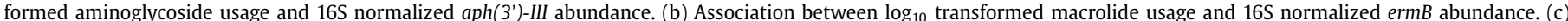

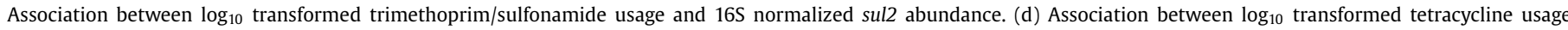
and $16 \mathrm{~S}$ normalized tetW abundance.

Table 1

Associations between antimicrobial usage (AMU), veal calf farm characteristics and relative abundance of aph(3')-III, ermB, sul2 and tetW resistance genes

\begin{tabular}{|c|c|c|c|c|c|c|c|c|c|c|}
\hline \multirow{2}{*}{$\begin{array}{l}\text { AMU } \\
\text { Aminoglycosides used (ref: no) }\end{array}$} & \multirow{2}{*}{$\begin{array}{l}\text { IQR or } \% \\
14 \%\end{array}$} & \multirow{2}{*}{$\begin{array}{l}\text { median } \\
(25-75 p c t)\end{array}$} & \multicolumn{2}{|c|}{$\begin{array}{l}\text { GMR }(95 \% \mathrm{CI}) \\
\operatorname{aph}\left(3^{\prime}\right)-I I I\end{array}$} & \multicolumn{2}{|l|}{ ermB } & \multicolumn{2}{|l|}{ sul2 } & \multicolumn{2}{|l|}{ tetW } \\
\hline & & & 1.42 & $(0.91-2.20)$ & & & & & & \\
\hline Log $\mathrm{TI}_{\text {DDDvet }}$ macrolides & 0.52 & $\begin{array}{l}0.5 \\
(0.09-0.61)\end{array}$ & & & 1.07 & $(0.82-1.39)$ & & & & \\
\hline Log $\mathrm{TI}_{\text {DDDvet }}$ trimethoprim/sulfonamides & 0.49 & $0(0-0.49)$ & & & & & 1.37 & $(1.09-1.74)$ & & \\
\hline Log $\mathrm{TI}_{\mathrm{DDDvet}}$ tetracyclines & 0.53 & $\begin{array}{l}0.74 \\
(0.39-0.92)\end{array}$ & & & & & 1.23 & $(0.99-1.52)$ & 0.94 & $(0.75-1.17)$ \\
\hline \multicolumn{11}{|l|}{ Farm characteristics } \\
\hline Other animals present at the farm (ref: no) & $57.6 \%$ & - & 0.71 & $(0.51-0.99)$ & & & & & & \\
\hline Weight of calves at arrival $(\mathrm{kg})$ & 4.9 & $\begin{array}{l}48 \\
(45.3-50.2)\end{array}$ & 0.82 & $(0.71-0.95)$ & 0.72 & $(0.62-0.85)$ & & & & \\
\hline Number of calves at sampling & 785 & $\begin{array}{l}729 \\
(258-1043)\end{array}$ & & & & & 0.83 & $(0.64-1.06)$ & & \\
\hline \multicolumn{11}{|l|}{ Agents used for cleaning } \\
\hline No cleaning & $8.5 \%$ & - & & & 0.67 & $(0.34-1.32)$ & & & 0.75 & $(0.44-1.25)$ \\
\hline Water only & $22 \%$ & - & & & 0.5 & $(0.29-0.84)$ & & & 0.66 & $(0.44-0.97)$ \\
\hline Soaking agents & $6.8 \%$ & - & & & 1.21 & $(0.58-2.53)$ & & & 1.1 & $(0.65-1.88)$ \\
\hline Disinfectants & $45.8 \%$ & - & & & 0.94 & $(0.59-1.50)$ & & & 0.97 & $(0.69-1.37)$ \\
\hline Soaking agents and disinfectants & $16.9 \%$ & - & & & ref & . & & & ref & . \\
\hline
\end{tabular}

$\mathrm{IQR}$, interquartile range; GMR, geometric mean ratio; $\mathrm{TI}_{\mathrm{DDDvet}}$, mean treatment incidence based on defined daily doses administered; $\mathrm{CI}$, confidence interval.

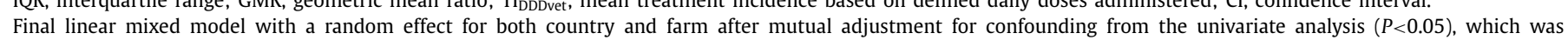
defined with the lowest Akaike's information criterion. Significant associations are marked in bold $(P<0.05)$.

association was found between the trim/sulfa use and the abundance of sul2 in the overall model. Similarly, one Danish study suggested a positive link between the faecal presence of sulfonamideresistant Escherichia coli in pigs and increased trim/sulfa administration [18]. However, for aminoglycoside, macrolide and tetracycline resistance, significant associations were not found between AMU and the respective ARG. An explanation for the absence of additional associations might be that antimicrobials administered in the early rearing phase do not affect faecal ARG levels in the period just before slaughter. In this study, a considerable number of farms reported no use of specific antimicrobial classes, while the corresponding ARGs could still be demonstrated in faecal samples of the respective farms. Therefore, it is hypothesized that actual AMU in veal calves only partially explains AMR, which is in line 
with results from European Union broiler farming that showed a similar resistome composition in untreated flocks compared with treated flocks [10].

A negative association was found between the weight of calves on arrival at the farm and faecal $\operatorname{aph}\left(3^{\prime}\right)$-III carriage and ermB carriage. A Swiss study also found a significantly higher AMR abundance among pigs of lower weight [19]. The weight of a calf on arrival at the farm could be an indicator of lower health status, resulting in the administration of a higher number of AMU treatments during its lifespan or a higher dosage of AMU upon arrival at the farm [7] to reduce the risk of infectious disease transmission.

Surprisingly, veal calves from farms that used only water for cleaning had significantly lower ermB and tetW concentrations in their faeces compared with calves from farms using both soaking agents and disinfectants. This is consistent with some previous studies in veal calves. In the study by Dorado-Garcia et al. [15], a specific cleaning and disinfecting programme was not effective to reduce the prevalence of MRSA in veal calf farms, while a positive association was observed between internal biosecurity (e.g. cleaning and disinfecting) and higher faecal AMR loads at pig farms [9]. It is difficult to explain the effect of cleaning agents on AMR against the background of significant differences in AMU in farms that use different cleaning agents. One hypothesis to explain this is that the application and residual action of biocides may contribute to co-selection of biocide-resistant genes and ARGs [20]. These results are, in themselves, not evidence against the use of disinfectants when cleaning livestock stables, but further studies to optimize cleaning and disinfection protocols in farms are advised.

\subsection{Strengths and limitations}

To the authors' knowledge, to date, this is the largest multicountry study on faecal AMR loads in veal calves [59 farms (405 samples)]. Despite this, bias and errors could have been introduced in this study. In general, non-differential exposure misclassification might lead to attenuation of associations. Another limitation is whether unknown historical AMU may have had a potential effect on AMR in the non-use farms. Finally, possible false-positive chance findings may not have been avoided completely.

\section{Conclusions}

A positive association was found between the use of trim/sulfa and the abundance of sul2 in faeces from veal calves. A higher weight of calves on arrival at the farm was negatively associated with $\operatorname{aph}\left(3^{\prime}\right)$-III and ermB. The use of only water for cleaning stables, compared with the use of soaking agents and disinfectants, showed a negative association with the abundance of AMR in veal calves.

\section{Acknowledgements}

The authors wish to thank all the field workers, laboratory analysts and data analysts at the Institute for Risk Assessment Sciences, the Netherlands (Daisy de Vries, Nynke Jansen, Janne Heederik, Jie Chen), the Stiftung Tierärztliche Hochschule Hannover, Germany (Maximiliane Brandt, Franziska Nienhaus) and the French Agency for Food, Environmental and Occupational Health and Safety, France (Florent Eono, Pamela Houée, Eric Eveno).

Members of the EFFORT consortium: Haitske Graveland (UUVM), Steven Sarrazin (UGENT), Alieda van Essen (WBVR), Bruno Gonzalez-Zorn (UCM), Gabriel Moyano (UCM), Pascal Sanders (ANSES), Julie David (ANSES), Antonio Battisti (IZSLT), Andrea Caprioli (IZSLT), Thomas Blaha (TIHO), Maximiliane Brandt (TIHO), Tine Hald (DTU), Ana Sofia Ribeiro Duarte (DTU), Magdalena Zajac (NVRI), Andrzej Hoszowski (deceased) (NVRI), Hristo
Daskalov (NDRVI), Helmut W. Saatkamp (BEC), Katharina D.C. Stärk (SAFOSO).

Funding: This work was part of the EFFORT project, co-funded by the European Commission, 7th Framework Programme for Research and Innovation (FP7-KBBE-2013-7, Grant No. 613754). Research at the National Veterinary Research Institute was also supported by a donation from the Polish Ministry of Science (Grant No. 3173/7PR/2014/2). This study also received financial support from the China Scholarships Council (Grant No. 201709110149).

Competing interests: None declared.

Ethical approval: Not required.

\section{References}

[1] World Health Organization Antimicrobial resistance: global report on surveillance. Geneva: WHO 2014:43-55

[2] McEwen SA, Fedorka-Cray PJ. Antimicrobial use and resistance in animals. Clin Infect Dis 2002;34(Suppl. 3):S93-106.

[3] Lava M, Schüpbach-Regula G, Steiner A, Meylan M. Antimicrobial drug use and risk factors associated with treatment incidence and mortality in Swiss veal calves reared under improved welfare conditions. Prev Vet Med 2016;126:121-30

[4] Chase CC, Hurley DJ, Reber AJ. Neonatal immune development in the calf and its impact on vaccine response. Vet Clin N Am Food Anim Pract 2008;24:87-104.

[5] Netherlands Veterinary Medicines Institute. Usage of Antibiotics in Agricultural Livestock in the Netherlands in 2018. Utrecht: SDa; 2019. p. 16-34.

[6] Van Gompel L, Luiken REC, Sarrazin S, Munk P, Knudsen BE, Hansen RB, et al. The antimicrobial resistome in relation to antimicrobial use and biosecurity in pig farming, a metagenome-wide association study in nine European countries. J Antimicrob Chemother 2019;74(4):865-76.

[7] Di Labio E, Regula G, Steiner A, Miserez R, Thomann A, Ledergerber U. Antimicrobial resistance in bacteria from Swiss veal calves at slaughter. Zoonoses Publ Health 2007;54:344-52.

[8] Graveland H, Wagenaar JA, Verstappen KM, Oosting-van Schothorst I, Heederik DJJ, Bos ME. Dynamics of MRSA carriage in veal calves: a longitudinal field study. Prev Vet Med 2012;107:180-6.

[9] Merle R, Hajek P, Käsbohrer A, Hegger-Gravenhorst C, Mollenhauer Y, Robanus M, et al. Monitoring of antibiotic consumption in livestock: a German feasibility study. Prev Vet Med 2012;104:34-43.

[10] Luiken RE, Van Gompel L, Munk P, Sarrazin S, Joosten P, Dorado-García A, et al. Associations between antimicrobial use and the faecal resistome on broiler farms from nine European countries. J Antimicrob Chemother 2019;74(9):2596-604.

[11] Ceccarelli D, Hesp A, van der Goot J, Joosten P, Sarrazin S, Wagenaar JA, et al. Antimicrobial resistance prevalence in commensal Escherichia coli from broilers, fattening turkeys, fattening pigs and veal calves in European countries and association with antimicrobial usage at country level. J Med Microbiol 2020;69(4):537-47.

[12] Van Gompel L, Dohmen W, Luiken REC, Bouwknegt M, Heres L, Van Heijnsbergen E, et al. Occupational exposure and carriage of antimicrobial resistance genes (tetW, ermB) in pig slaughterhouse workers. Ann Work Expos Health 2020;64(2):125-37.

[13] Woegerbauer M, Zeinzinger J, Springer B, Hufnagl P, Indra A, Korschineck I, et al. Prevalence of the aminoglycoside phosphotransferase genes aph ( $\left.3^{\prime}\right)$-IIIa and aph (3')-IIa in Escherichia coli, Enterococcus faecalis, Enterococcus faecium, Pseudomonas aeruginosa, Salmonella enterica subsp. enterica and Staphylococcus aureus isolates in Austria. J Med Microbiol 2014;63:210-17.

[14] Heuer H, Focks A, Lamshöft M, Smalla K, Matthies M, Spiteller M. Fate of sulfadiazine administered to pigs and its quantitative effect on the dynamics of bacterial resistance genes in manure and manured soil. Soil Biol Biochem 2008;40:1892-900.

[15] Dorado-García A, Graveland H, Bos ME, Verstappen KM, Van Cleef BA, Kluytmans JA, et al. Effects of reducing antimicrobial use and applying a cleaning and disinfection program in veal calf farming: experiences from an intervention study to control livestock-associated MRSA. PLoS One 2015;10:e0135826.

[16] Wilkins M, Bartlett P, Berry D, Perry R, Fitzgerald S, Bernardo T, et al. Absence of Mycobacterium bovis infection in dogs and cats residing on infected cattle farms: Michigan, 2002. Epidemiol Infect 2008;136:1617-23.

[17] Aiello AE, Larson E. Antibacterial cleaning and hygiene products as an emerging risk factor for antibiotic resistance in the community. Lancet Infect Dis 2003;3:501-6.

[18] Hammerum AM, Sandvang D, Andersen SR, Seyfarth AM, Porsbo LJ, Frimodt-Møller N, et al. Detection of sul1, sul2 and sul3 in sulphonamide resistant Escherichia coli isolates obtained from healthy humans, pork and pigs in Denmark. Int J Food Microbiol 2006;106:235-7.

[19] Schuppers M, Stephan R, Ledergerber U, Danuser J, Bissig-Choisat B, Stärk K, et al. Clinical herd health, farm management and antimicrobial resistance in Campylobacter coli on finishing pig farms in Switzerland. Prev Vet Med 2005;69:189-202

[20] Costa SS, Viveiros M, Amaral L, Couto L. Multidrug efflux pumps in Staphylococcus aureus: an update. Open Microbiol J 2013;7:59. 